\title{
High Frequency Correlation Modelling
}

\author{
Huth Nicolas ${ }^{1}$ and Abergel Frédéric, ${ }^{2}$ \\ ${ }^{1}$ Chair of Quantitative Finance, Ecole Centrale Paris nicolas.hutheecp. fr \\ ${ }^{2}$ Chair of Quantitative Finance, Ecole Centrale Paris frederic.abergel@ecp. fr
}

Many statistical arbitrage strategies, such as pair trading or basket trading, are based on several assets. Optimal execution routines should also take into account correlation between stocks when proceeding clients orders. However, not so much effort has been devoted to correlation modelling and only few empirical results are known about high frequency correlation. Depending on the time scale under consideration, a plausible candidate for modelling correlation should:

- at high frequency: reproduce the Epps effect [1], take into account lead-lag relationships between assets [2]

- at the daily scale: avoid purely Gaussian correlations [3]

We develop a theoretical framework based on correlated point processes in order to capture the Epps effect in section 1. We show in section 2 that this model converges to correlated Brownian motions when moving to large time scales. A way of introducing non-Gaussian correlations is also discussed in section 2. We conclude by addressing the limits of this model and further research on high frequency correlation.

\section{A model for high frequency correlation}

In this section, we start by reviewing the most famous empirical fact about high frequency correlation, namely the Epps effect. Then we suggest a theoretical framework that captures this salient feature of high frequency data.

\subsection{Empirical fact: the Epps effect}

In 1979, T.W. Epps[1] observed that, in his own words:

"Correlations among price changes [...] are found to decrease with the length of the interval for which the price changes are measured." 
This result was then recovered on more recent data and on several markets $[4,5$, $6,7]$. Two reasons for the Epps effect were advocated in the literature:

- Market orders on two assets are asynchronous: as $\Delta t \rightarrow 0$

$$
P\left(\left(\left\{X_{i \Delta t}-X_{(i-1) \Delta t}\right) \neq 0\right\} \cap\left\{\left(Y_{i \Delta t}-Y_{(i-1) \Delta t}\right) \neq 0\right\}\right) \rightarrow 0
$$

so that $\sum_{i}\left(X_{i \Delta t}-X_{(i-1) \Delta t}\right)\left(Y_{i \Delta t}-Y_{(i-1) \Delta t}\right) \rightarrow 0$, where $X_{i \Delta t}$ and $Y_{i \Delta t}$ are the prices of two assets recorded at time $i \Delta t$ ( $\Delta t$ being the sampling period) with any scheme of interpolation (previous-tick, linear, etc...). Indeed, when sampling prices at very high frequency, it is highly unlikely that both assets will experience a price jump.

- Information needs a human time scale to be processed [2]. On financial markets, some assets, called the leaders, which are often the most liquid, incorporate information onto their prices faster than others, called the laggers. As a result, when dealing with two assets that exhibit a so-called lead-lag relationship, there will be only partial correlation at timescales shorter than the characteristic lead-lag time.

Figure 1 illustrates the Epps effect on French high frequency data. The stocks studied are BNPP.PA, SOGN.PA, RENA.FP, VLOF.PA, LVMH.PA, LYOE.PA during the time period from 2008-02-04 to 2008-20-03. Clearly, the correlation is almost nil when sampling at few seconds of trading. The correlation converges to an asymptotic level after about half an hour of trading.

As a testimony that the Epps effect is not only due to asynchronicity, figure 2 plots the empirical probability of having both assets jumping in a time window of a given length for the same three pairs of stocks. Obviously, this probability is quite small for few seconds. It is noteworthy that the characteristic time of this probability is not only smaller than the Epps effect one but is also the same for all the three pairs of stocks. If asynchronicity was to be the sole reason for the Epps effect, then all the Epps curves should converge after about ten minutes.

\subsection{Correlated point processes as a model for correlation}

Because of the existence of a minimal price change on markets, called the tick, prices are closer to pure jump processes rather than diffusions, which is the standard assumption for daily data. When looking at several assets, a natural question that arises is therefore how to correlate point processes. As a point process is fully described by either the associated counting process or the intensity process, there are basically four ways of addressing the issue of correlation, that we explain in table 1.

From the microstructure of markets point of view, the upper left solution is unrealistic because it is highly unlikely that two market orders on two different assets are executed at the very same time. Therefore, the most appropriate solution is to use correlated stochastic intensities. The upper right solution, i.e. Hawkes processes, can be interpreted as particular choice for the correlation structure: past events on one asset 


\section{Epps effect}

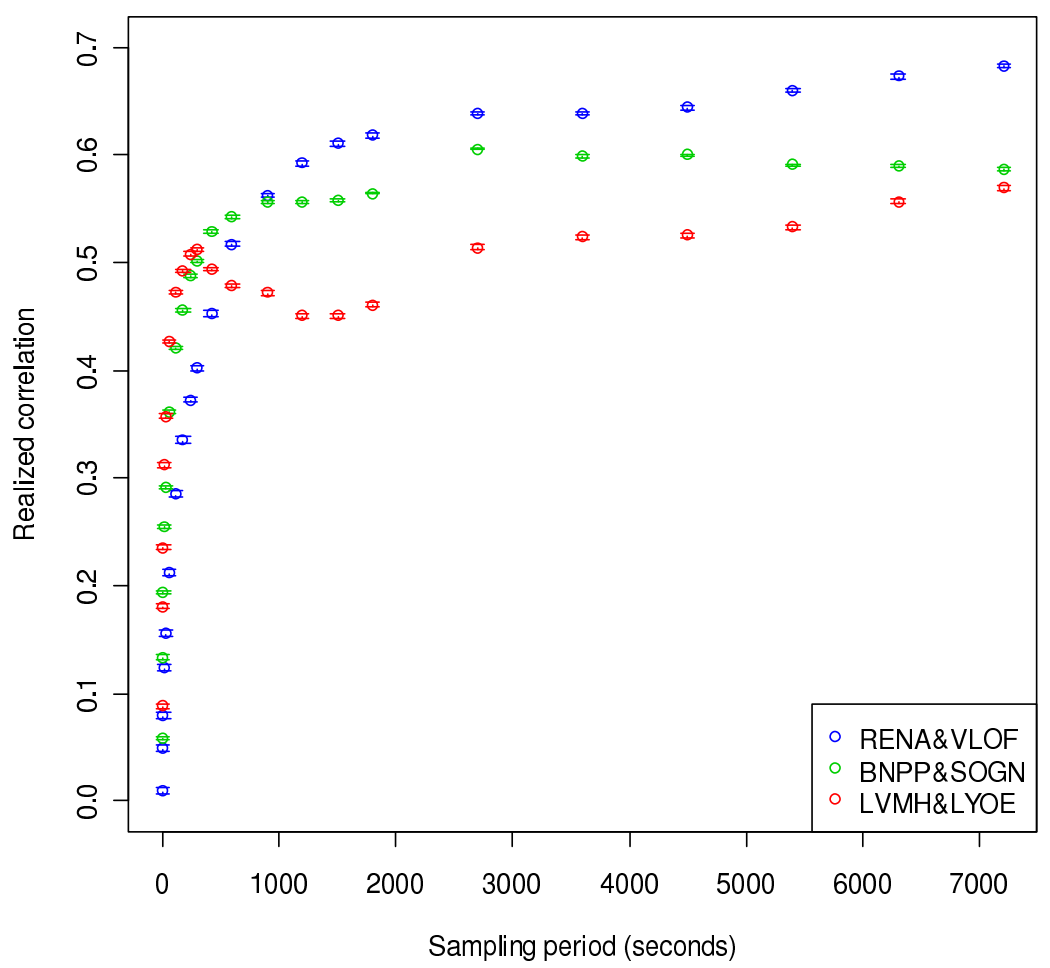

Fig. 1. Correlation coefficient for three pairs of French stocks as a function of the sampling period of price changes.

Table 1. Four ways of correlating two point processes

\begin{tabular}{|c|c|c|}
\hline & $\mathrm{N}_{2}$ & $\lambda_{2}$ \\
\hline $\mathrm{N}_{1}$ & sync. jumps & Hawkes processes \\
& $\left(\right.$ ex: $\left.N_{2}=N_{1}+N_{0}\right)$ & $\lambda_{2}=f\left(N_{1}, \ldots\right)$ \\
\hline$\lambda_{1}$ & & async. jumps \\
& & $\lambda$ 's must be random \\
\hline
\end{tabular}

tend to trigger (or inhibit) events on the other asset, so that correlation is endogenous.

Let us consider the following model:

$$
\mathrm{d} P_{t}^{i}=\Delta P^{i}\left(\mathrm{~d} N_{t}^{i,+}-\mathrm{d} N_{t}^{i,-}\right)
$$

where

- $\quad P_{t}^{i}$ is the price of asset $i$ at time $t$. It can be all kind of prices: last traded price, midprice, best limit, etc... 
Occurence of synch. jumps

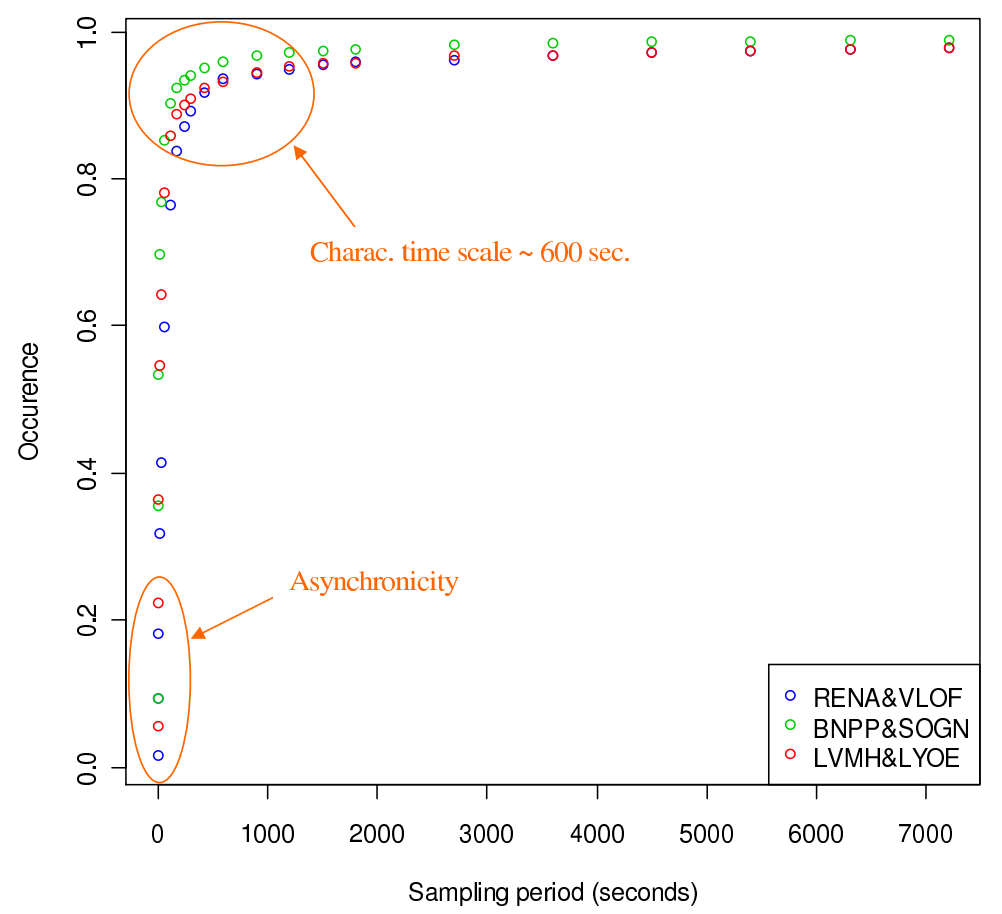

Fig. 2. Empirical probability of having both prices jumping in a time window of a given length as a function of this length for three pairs of French stocks.

- $\quad \Delta P^{i}$ is the jump size for asset $i$. It is assumed to be constant for the sake of simplicity.

- $N_{t}^{i,+}$ (resp. $N_{t}^{i,-}$ ) is the number of market orders that triggered upward (resp. downward) price changes for asset $i$ up to time $t$.

This model is a pure price model in the sense that it does not explicitly take into account the dynamics of the order book, which impacts $\Delta P^{i}$. In this model, we can explicitly compute the Epps curve ${ }^{3}$ :

$$
\rho_{\Delta t}=\operatorname{Corr}\left(\mathrm{d} P_{\Delta t}^{1}, \mathrm{~d} P_{\Delta t}^{2}\right)=\frac{a}{\sqrt{b_{0}+\frac{b_{1}}{\Delta t}+\frac{b_{2}}{\Delta t^{2}}}}
$$

where

\footnotetext{
${ }^{3}$ See appendix A for the proof.
} 


$$
\begin{aligned}
& a=\operatorname{Cov}\left(\lambda^{1,+}-\lambda^{1,-}, \lambda^{2,+}-\lambda^{2,-}\right) \\
& b_{0}=\operatorname{Var}\left(\lambda^{1,+}-\lambda^{1,-}\right) \operatorname{Var}\left(\lambda^{2,+}-\lambda^{2,-}\right) \\
& b_{1}=E\left(\lambda^{1,+}+\lambda^{1,-}\right) \operatorname{Var}\left(\lambda^{2,+}-\lambda^{2,-}\right)+E\left(\lambda^{2,+}+\lambda^{2,-}\right) \operatorname{Var}\left(\lambda^{1,+}-\lambda^{1,-}\right) \\
& b_{2}=E\left(\lambda^{1,+}+\lambda^{1,-}\right) E\left(\lambda^{2,+}+\lambda^{2,-}\right)
\end{aligned}
$$

The features of $\rho_{\Delta t}$ are:

- $\rho_{\Delta t} \rightarrow 0$ as $\Delta t \rightarrow 0$

- $\rho_{\Delta t} \rightarrow \frac{a}{\sqrt{b_{0}}}=\operatorname{Corr}\left(\lambda^{1,+}-\lambda^{1,-}, \lambda^{2,+}-\lambda^{2,-}\right)$ as $\Delta t \rightarrow+\infty$

- $\rho_{\Delta t}$ is increasing and concave

which reproduce most of the curves plotted on figure 1. In the case of timedependent intensities $\left(\lambda_{t}^{1, \pm}, \lambda_{t}^{2, \pm}, t \geq 0\right)$, we get

$$
\begin{gathered}
\rho_{\Delta t}=\operatorname{Corr}\left(\mathrm{d} P_{\Delta t}^{1}, \mathrm{~d} P_{\Delta t}^{2}\right)=\frac{a_{\Delta t}}{\sqrt{b_{0, \Delta t}+\frac{b_{1, \Delta t}}{\Delta t}+\frac{b_{2, \Delta t}}{\Delta t^{2}}}} \\
a_{\Delta t}=\operatorname{Cov}\left(\frac{1}{\Delta t} \int_{0}^{\Delta t}\left(\lambda_{s}^{1,+}-\lambda_{s}^{1,-}\right) \mathrm{d} s, \frac{1}{\Delta t} \int_{0}^{\Delta t}\left(\lambda_{s}^{2,+}-\lambda_{s}^{2,-}\right) \mathrm{d} s\right) \\
b_{0, \Delta t}=\operatorname{Var}\left(\frac{1}{\Delta t} \int_{0}^{\Delta t}\left(\lambda_{s}^{1,+}-\lambda_{s}^{1,-}\right) \mathrm{d} s\right) \operatorname{Var}\left(\frac{1}{\Delta t} \int_{0}^{\Delta t}\left(\lambda_{s}^{2,+}-\lambda_{s}^{2,-}\right) \mathrm{d} s\right) \\
b_{1, \Delta t}=E\left(\frac{1}{\Delta t} \int_{0}^{\Delta t}\left(\lambda_{s}^{1,+}+\lambda_{s}^{1,-}\right) \mathrm{d} s\right) \operatorname{Var}\left(\frac{1}{\Delta t} \int_{0}^{\Delta t}\left(\lambda_{s}^{2,+}-\lambda_{s}^{2,-}\right) \mathrm{d} s\right) \\
+E\left(\frac{1}{\Delta t} \int_{0}^{\Delta t}\left(\lambda_{s}^{2,+}+\lambda_{s}^{2,-}\right) \mathrm{d} s\right) \operatorname{Var}\left(\frac{1}{\Delta t} \int_{0}^{\Delta t}\left(\lambda_{s}^{1,+}-\lambda_{s}^{1,-}\right) \mathrm{d} s\right) \\
b_{2, \Delta t}=E\left(\frac{1}{\Delta t} \int_{0}^{\Delta t}\left(\lambda_{s}^{1,+}+\lambda_{s}^{1,-}\right) \mathrm{d} s\right) E\left(\frac{1}{\Delta t} \int_{0}^{\Delta t}\left(\lambda_{s}^{2,+}+\lambda_{s}^{2,-}\right) \mathrm{d} s\right)
\end{gathered}
$$

In particular, the use of time-dependent intensities allows for non-monotonous shapes of $\rho_{\Delta t}$.

Figure 3 shows least squares fits of the empirical curves with equation 3 . The last fit is not as accurate as the two firsts because the Epps curve is not monotonous. This is the typical case where time-dependent intensities are needed to achieve a better fit.

The least square fit is not really natural and can not allow us to decide whether our model is sophisticated enough to reproduce market mechanisms or not. We should rather use maximum likelihood or moments estimation. If our model is right, then the asymptotic value of the correlation should be equal to $\operatorname{Corr}\left(\lambda^{1,+}-\lambda^{1,-}, \lambda^{2,+}-\lambda^{2,-}\right)$, that we approximate by the empirical correlation of the imbalances of upwards/downwards 
BNPP, SOGN
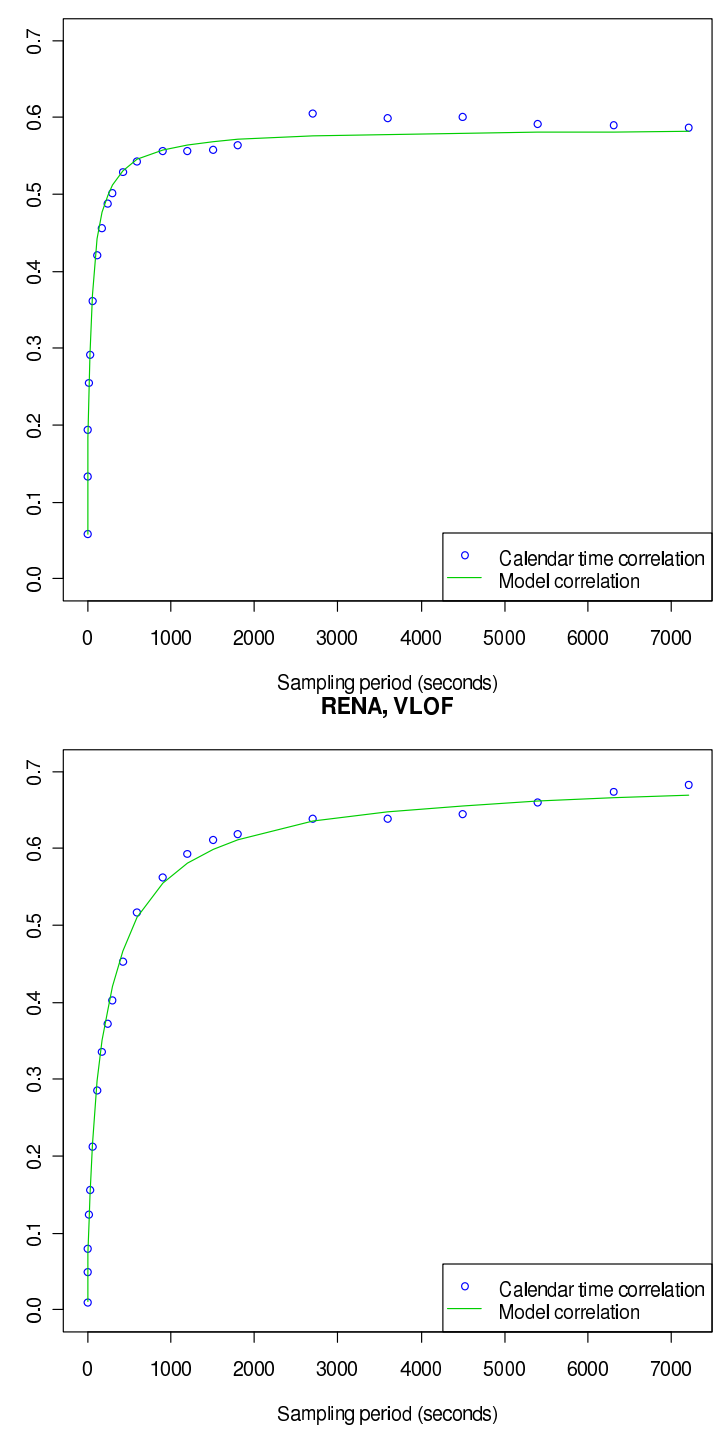

LVMH, LYOE

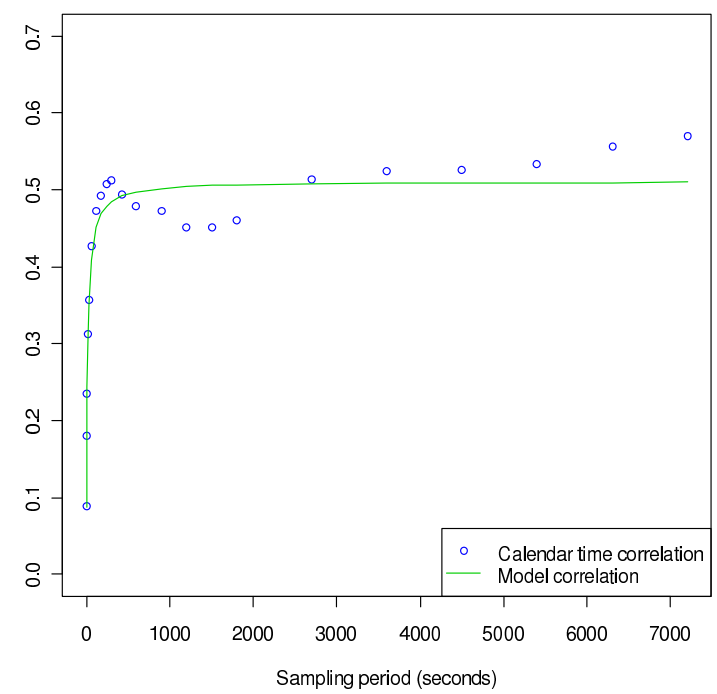

Fig. 3. Least squares fit of equation 3 for three pairs of French stocks. 
moves $\operatorname{Corr}\left(N^{1,+}-N^{1,-}, N^{2,+}-N^{2,-}\right)$. We also compute the correlation of the imbalances of buy/sell market orders and the correlation of the total number of trades $\operatorname{Corr}\left(N^{1,+}+N^{1,-}, N^{2,+}+N^{2,-}\right)$ for comparison. Figure 4 shows how these quantities behave.

The asymptotic level of the empirical correlation is rather far from what the model predicts, i.e. the upwards/downwards imbalance correlation. So there might be other market mechanisms that are important to take into account. In particular, it seems that the correlation of returns is close to the correlation of the total number of trades $\operatorname{Corr}\left(N^{1,+}+N^{1,-}, N^{2,+}+N^{2,-}\right)$, which is a good proxy for the correlation of the variances of returns $\operatorname{Corr}\left(\left(\mathrm{d} P^{1}\right)^{2},\left(\mathrm{~d} P^{2}\right)^{2}\right)$.

\section{Large scale limit}

So far we have been concerned with high frequency correlation modelling. We might be interested in how a model behaves after a long time, a day, say. More stylized facts are known about daily time series[3]. In particular, we would like our model on a daily scale to

- exhibit a diffusive behavior: $\operatorname{Var}\left(\mathrm{d} P_{\Delta t}\right) \propto \Delta t$

- display non-Gaussian tails for the distribution of returns

- reproduce volatility clustering

- allow for a non-Gaussian correlation structure, such as extreme correlations

In order to study the large scale limit of the point process model, let us consider the special case of Hawkes processes, which was introduced in [8]

$$
\begin{aligned}
& \mathrm{d} P_{t}^{i,(\delta)}=\Delta P^{i} \sqrt{\delta}\left(\mathrm{d} N_{t / \delta}^{i,+}-\mathrm{d} N_{t / \delta}^{i,-}\right) \\
& \lambda_{t}^{i, \pm}=\mu+\int_{0}^{t} \phi(t-s) \mathrm{d} N_{s}^{i, \mp}+\int_{0}^{t} \psi(t-s) \mathrm{d} N_{s}^{j, \pm}
\end{aligned}
$$

with the stationarity constraint that the spectral radius of the matrix of the $L^{1}$ norm of regression kernels is strictly less than one. The large scale limit is reached as $\delta \rightarrow 0$. It can be shown ${ }^{4}$ that this model converges to correlated Brownian motions

\footnotetext{
${ }^{4}$ See appendix B for the proof.
} 

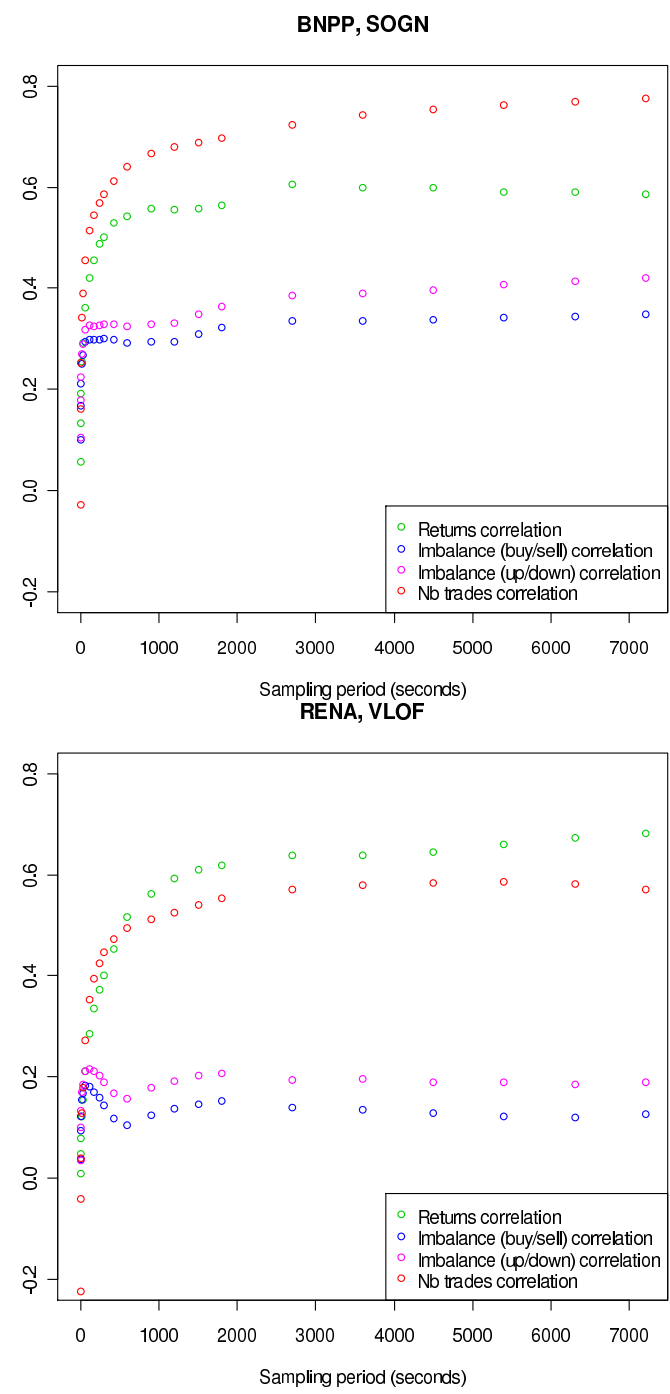

LVMH, LYOE

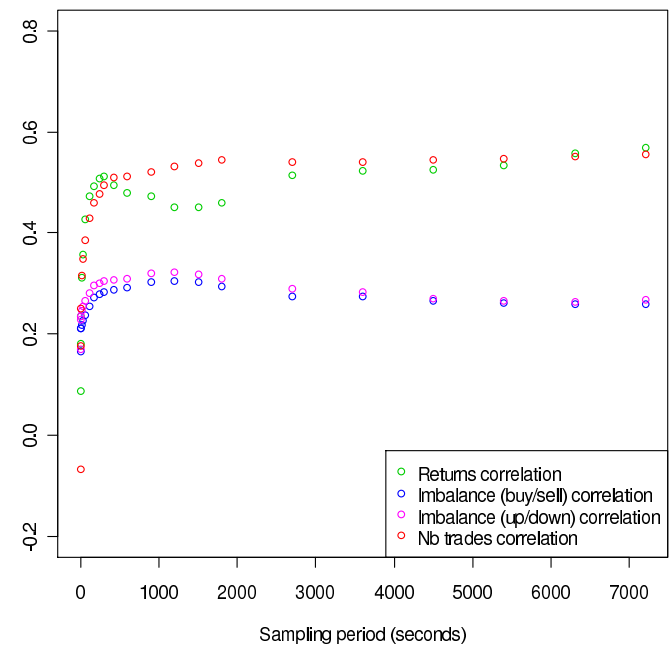

Fig. 4. Comparison between various correlations for three pairs of French stocks. 


$$
\begin{aligned}
& \mathrm{d} P_{t}^{i, 0}=\sigma \cdot \Delta P_{i} \cdot C\left(\mathrm{~d} B_{t}^{i}+D \cdot \mathrm{d} B_{t}^{j}\right) \\
& \sigma:=\sqrt{\frac{2 \mu}{1-(\|\phi\|+\|\psi\|)}} \\
& C:=\frac{1+\|\phi\|}{(1+\|\phi\|)^{2}-\|\psi\|^{2}} \\
& D:=\frac{\|\psi\|}{1+\|\phi\|}
\end{aligned}
$$

where $\left(B^{1}, B^{2}\right)$ is a standard bi-dimensional Brownian motion. So this model achieves a diffusive behaviour but fails to reproduce non-Gaussian tails and nonGaussian correlation and volatility clustering.

We suggest a way of making the correlation structure more complex by introducing a common exogenous noise in the dynamics of the intensities

$$
\begin{aligned}
& \mathrm{d} P_{t}^{i, \delta}=\Delta P_{i} \sqrt{\delta}\left(\mathrm{d} N_{t / \delta}^{i,+}-\mathrm{d} N_{t / \delta}^{i,-}\right) \\
& \lambda_{t}^{i, \pm}=\mu+\int_{0}^{t} \phi(t-s) \mathrm{d} N_{s}^{i, \mp}+\int_{0}^{t} \psi(t-s) \mathrm{d} N_{s}^{j, \pm}+M_{t}^{ \pm}
\end{aligned}
$$

Then the diffusive limit reads

$$
\begin{aligned}
\mathrm{d} P_{t}^{i, 0}=\Delta P_{i} \cdot C\left(\sigma\left(\mathrm{d} B_{t}^{i}+D . \mathrm{d} B_{t}^{j}\right)+E \cdot \mathrm{d} M_{t}^{0}\right) \\
\sigma:=\sqrt{\frac{2 \mu}{1-(\|\phi\|+\|\psi\|)}} \\
C:=\frac{1+\|\phi\|}{(1+\|\phi\|)^{2}-\|\psi\|^{2}} \\
D:=\frac{\|\psi\|}{1+\|\phi\|} \\
E:=\frac{1+\|\phi\|+\|\psi\|}{1+\|\phi\|} \\
M_{t}^{0}:=M_{t}^{0,+}-M_{t}^{0,-}:=\lim _{\delta \rightarrow 0} \sqrt{\delta} \int_{0}^{t / \delta}\left(M_{s}^{+}-M_{s}^{-}\right) \mathrm{d} s
\end{aligned}
$$

As a result, the correlation coefficient reads

$$
\rho=\frac{2 \sigma^{2} D+E^{2} \frac{\mathrm{d}\left\langle M^{0}\right\rangle_{t}}{\mathrm{~d} t}}{\sigma^{2}\left(1+D^{2}\right)+E^{2} \frac{\mathrm{d}\left\langle M^{0}\right\rangle_{t}}{\mathrm{~d} t}}
$$


which is stochastic as long as $\frac{\mathrm{d}\left\langle M^{0}\right\rangle_{t}}{\mathrm{~d} t}$ is ${ }^{5}$. For instance, $M^{0}$ can be interpreted as a market driver which is common to all stocks. Therefore the correlation depends on the market volatility. Indeed, many studies on daily correlation matrices tend to show that the largest part of the spectra of the correlation is explained by a market factor, rather than by direct interactions between stocks.

\section{Conclusion and further research}

We studied a framework for high frequency correlation modelling based on point processes. This model exhibits a correlation structure that depends on the time scale, in agreement with the Epps effect. However, it seems that there are still market mechanisms to be included to make it more in agreement with real data.

There are still open questions regarding high frequency correlation such as leadlag estimation $[9,10]$ and modelling. Furthermore, the role played by the order book shape in the dynamics of correlation has never been studied. We are also investigating ways of achieving better fits of empirical data by adding new parameters in the dynamics of the intensities of market orders, such as an exogenous noise.

\section{References}

1. Epps T. W. (1979) Comovements in Stock Prices in the Very Short-Run. Journal of the American Statistical Association 74, 291-298

2. Toth B., Kertesz J. (2009) The Epps Effect Revisited, Quantitative Finance 9(7), 793-802

3. Bouchaud J. -P., Potters M. (2004) Theory of Financial Risk and Derivative Pricing, From Statistical Physics to Risk Management. Cambridge University Press

4. Reno R. (2003) A Closer Look at the Epps Effect. International Journal of Theoretical and Applied Finance 6: 87-102

5. Toth B., Kertesz J. (2006) Increasing Market Efficiency: Evolution of Cross-Correlations of Stock Returns, Physica A 360, 505-515

6. Iori G., Precup O.V. (2006) Cross-correlation Measures in the High Frequency Domain, Working Paper

7. Toth B., Kertesz J. (2007) On the origin of the Epps Effect, Physica A 383(1), 54-58

8. Bacry E. (2010) Modeling microstructure noise using point processes, EconophysKolkata V Conference

9. Robert C. Y., Rosenbaum M. (2009) On the limiting spectral distribution of the covariance matrices of time-lagged processes, to appear in Journal of Multivariate Analysis

10. Robert C. Y., Rosenbaum M., Hoffman M., Yoshida N. (2010) Estimation of the lead-lag parameter from non-synchronous data, Working Paper

\footnotetext{
${ }^{5}$ The brackets stand for the quadratic variation of a stochastic process.
} 


\section{Appendix A}

Standard computations show that

$$
\begin{array}{r}
\operatorname{Cov}\left(\mathrm{d} P_{\Delta t}^{1}, \mathrm{~d} P_{\Delta t}^{2}\right)=\Delta P^{1} \Delta P^{2}\left(\operatorname{Cov}\left(N_{\Delta t}^{1,+}, N_{\Delta t}^{2,+}\right)+\operatorname{Cov}\left(N_{\Delta t}^{1,-}, N_{\Delta t}^{2,-}\right)\right. \\
\left.-\operatorname{Cov}\left(N_{\Delta t}^{1,+}, N_{\Delta t}^{2,-}\right)-\operatorname{Cov}\left(N_{\Delta t}^{1,-}, N_{\Delta t}^{2,+}\right)\right) \\
\operatorname{Var}\left(\mathrm{d} P_{\Delta t}^{i}\right)=\left(\Delta P^{i}\right)^{2}\left(\operatorname{Var}\left(N_{\Delta t}^{i,+}\right)+\operatorname{Var}\left(N_{\Delta t}^{i,-}\right)-2 \operatorname{Cov}\left(N_{\Delta t}^{i,+}, N_{\Delta t}^{i,-}\right)\right)
\end{array}
$$

and

$$
\begin{gathered}
\operatorname{Cov}\left(N_{\Delta t}^{1, a}, N_{\Delta t}^{2, b}\right)=E\left(\operatorname{Cov}\left(N_{\Delta t}^{1, a}, N_{\Delta t}^{2, b}\right) \mid \lambda^{1, a}, \lambda^{2, b}\right)+\operatorname{Cov}\left(E\left(N_{\Delta t}^{1, a} \mid \lambda^{1, a}\right), E\left(N_{\Delta t}^{2, b} \mid \lambda^{2, b}\right)\right) \\
=0+\Delta t^{2} \cdot \operatorname{Cov}\left(\lambda^{1, a}, \lambda^{2, b}\right) \\
\text { for } a, b \in\{+,-\} \text { and } \\
\qquad \operatorname{Var}\left(N_{\Delta t}^{i, \pm}\right)=\Delta t . E\left(\lambda^{i, \pm}\right)+\Delta t^{2} \cdot \operatorname{Var}\left(\lambda^{i, \pm}\right)
\end{gathered}
$$

Finally, we get

$$
\operatorname{Corr}\left(\mathrm{d} P_{\Delta t}^{1}, \mathrm{~d} P_{\Delta t}^{2}\right)=\frac{a}{\sqrt{b_{0}+\frac{b_{1}}{\Delta t}+\frac{b_{2}}{\Delta t^{2}}}}
$$

where

$$
\begin{aligned}
& a=\operatorname{Cov}\left(\lambda^{1,+}-\lambda^{1,-}, \lambda^{2,+}-\lambda^{2,-}\right) \\
& b_{0}=\operatorname{Var}\left(\lambda^{1,+}-\lambda^{1,-}\right) \operatorname{Var}\left(\lambda^{2,+}-\lambda^{2,-}\right) \\
& b_{1}=E\left(\lambda^{1,+}+\lambda^{1,-}\right) \operatorname{Var}\left(\lambda^{2,+}-\lambda^{2,-}\right)+E\left(\lambda^{2,+}+\lambda^{2,-}\right) \operatorname{Var}\left(\lambda^{1,+}-\lambda^{1,-}\right) \\
& b_{2}=E\left(\lambda^{1,+}+\lambda^{1,-}\right) E\left(\lambda^{2,+}+\lambda^{2,-}\right)
\end{aligned}
$$

so that

$$
\begin{aligned}
\lim _{\Delta t \rightarrow 0} \operatorname{Corr}\left(\mathrm{d} P_{\Delta t}^{1}, \mathrm{~d} P_{\Delta t}^{2}\right) & =0 \\
\lim _{\Delta t \rightarrow+\infty} \operatorname{Corr}\left(\mathrm{d} P_{\Delta t}^{1}, \mathrm{~d} P_{\Delta t}^{2}\right) & =\frac{a}{\sqrt{b_{0}}}=\operatorname{Corr}\left(\lambda^{1,+}-\lambda^{1,-}, \lambda^{2,+}-\lambda^{2,-}\right)
\end{aligned}
$$

In the case of time-dependent intensities, the same line of computing goes except that $\lambda$ 's have to be replaced by their time average $\frac{1}{\Delta t} \int_{t}^{t+\Delta t} \lambda(s) \mathrm{d} s$. 


\section{Appendix B}

Let us consider the following Hawkes model

$$
\begin{aligned}
& \mathrm{d} P_{t}=\Delta P\left(\mathrm{~d} N_{t}^{+}-\mathrm{d} N_{t}^{-}\right) \\
& \lambda_{t}^{ \pm}=\mu+\int_{0}^{t} \phi(t-s) \mathrm{d} N_{s}^{\mp}
\end{aligned}
$$

where $\Delta P \in R^{+}$and $\|\phi\|<1$. We are looking for the diffusive limit of this model. Therefore, we introduce the rescaled model

$$
\mathrm{d} P_{t}^{\delta}=\Delta P \sqrt{\delta}\left(\mathrm{d} N_{t / \delta}^{+}-\mathrm{d} N_{t / \delta}^{-}\right)
$$

We are interested in the limit $\delta \rightarrow 0$. Let us split the price into two parts: the martingale and the compensator

$$
\begin{aligned}
& \mathrm{d} P_{t}^{\delta}=\mathrm{d} M_{t}^{\delta}+\mathrm{d} C_{t}^{\delta} \\
& M_{t}^{\delta}=\Delta P \sqrt{\delta}\left(\left(N_{t / \delta}^{+}-N_{t / \delta}^{-}\right)-\int_{0}^{t / \delta}\left(\lambda_{s}^{+}-\lambda_{s}^{-}\right) \mathrm{d} s\right) \\
& C_{t}^{\delta}=\Delta P \sqrt{\delta} \int_{0}^{t / \delta}\left(\lambda_{s}^{+}-\lambda_{s}^{-}\right) \mathrm{d} s
\end{aligned}
$$

Regarding the compensator, we have

$$
\begin{aligned}
C_{t}^{\delta} & =\Delta P \sqrt{\delta} \int_{0}^{t / \delta}\left(\lambda_{s}^{+}-\lambda_{s}^{-}\right) \mathrm{d} s \\
& =-\|\phi\| \int_{0}^{t} \mathrm{~d} P_{u}^{\delta} \int_{0}^{\frac{t-u}{\delta}} \frac{\phi(x)}{\|\phi\|} \mathrm{d} x \rightarrow-\|\phi\| \mathrm{d} P_{t}^{0}
\end{aligned}
$$

Let us compute the quadratic variation of the martingale part

$$
\begin{aligned}
\frac{\left\langle M^{\delta}\right\rangle_{t}}{\Delta P^{2}} & =\delta \int_{0}^{t / \delta}\left(\lambda_{s}^{+}+\lambda_{s}^{-}\right) \mathrm{d} s \\
& =2 \mu t+\delta \int_{0}^{t / \delta}\left(\mathrm{d} N_{u}^{+}+\mathrm{d} N_{u}^{-}\right) \int_{0}^{t / \delta-u} \phi(x) \mathrm{d} x \\
& =2 \mu t+\delta \int_{0}^{t / \delta} \mathrm{d} N_{u}^{+}-\lambda_{u}^{+} \mathrm{d} u+\mathrm{d} N_{u}^{-}-\lambda_{u}^{-} \mathrm{d} u \int_{0}^{t / \delta-u} \phi(x) \mathrm{d} x+\delta \int_{0}^{t / \delta}\left(\lambda_{u}^{+}+\lambda_{u}^{-}\right) \mathrm{d} u \int_{0}^{t / \delta-u} \phi(x) \mathrm{d} x \\
& \rightarrow 2 \mu t \sum_{n \geq 0}\|\phi\|^{n}=\left(\frac{2 \mu}{1-\|\phi\|}\right) t
\end{aligned}
$$


since

$\lim _{\delta \rightarrow 0} \delta \int_{0}^{t / \delta}\left(\left(\mathrm{d} N_{u}^{+}-\lambda_{u}^{+} \mathrm{d} u\right)+\left(\mathrm{d} N_{u}^{-}-\lambda_{u}^{-} \mathrm{d} u\right)\right) \int_{0}^{t / \delta-u} \phi(x) \mathrm{d} x=\lim _{\delta \rightarrow 0} \sqrt{\delta}\|\phi\|\left(B_{t}^{+}+B_{t}^{-}\right)=0$

Therefore, we get $M_{t}^{\delta} \rightarrow \sqrt{\frac{2 \mu}{1-\|\phi\|}}(\Delta P) B_{t}$ by using the following lemma.

Lemma 1. Let $M$ be a local martingale and $<M>_{\infty}:=\lim _{t \rightarrow+\infty}<M>_{t}$. Then

$$
E\left(\sup _{t \geq 0} M_{t}^{2}\right) \leq 4 \cdot E\left(<M>_{\infty}\right)
$$

If these two quantities are finite, then $M$ is a martingale which converges a.s. and in $L^{2}$ towards a random variable $M_{\infty}$ as $t \rightarrow+\infty$.

Finally, the diffusive limit reads

$$
\mathrm{d} P_{t}^{0}=\sqrt{\frac{2 \mu}{1-\|\phi\|}} \frac{\Delta P}{1+\|\phi\|} \mathrm{d} B_{t}
$$

In the case of a bivariate price model

$$
\begin{aligned}
& \mathrm{d} P_{t}^{i, \delta}=\Delta P_{i} \sqrt{\delta}\left(\mathrm{d} N_{t / \delta}^{i,+}-\mathrm{d} N_{t / \delta}^{i,-}\right) \\
& \lambda_{t}^{i, \pm}=\mu+\int_{0}^{t} \phi(t-s) \mathrm{d} N_{s}^{i, \mp}+\int_{0}^{t} \psi(t-s) \mathrm{d} N_{s}^{j, \pm}
\end{aligned}
$$

The very same line of proof yields

$$
\begin{aligned}
& \mathrm{d} P_{t}^{i, 0}=\sigma \cdot \Delta P_{i} \cdot C\left(\mathrm{~d} B_{t}^{i}+D \cdot \mathrm{d} B_{t}^{j}\right) \\
& \sigma:=\sqrt{\frac{2 \mu}{1-(\|\phi\|+\|\psi\|)}} \\
& C:=\frac{1+\|\phi\|}{(1+\|\phi\|)^{2}-\|\psi\|^{2}} \\
& D:=\frac{\|\psi\|}{1+\|\phi\|}
\end{aligned}
$$

so that the correlation coefficient of the two assets is constant

$$
\rho=\frac{\left\langle P_{t}^{1,0}, P^{2,0}\right\rangle_{t}}{\sqrt{\left\langle P^{1,0}\right\rangle_{t}\left\langle P^{2,0}\right\rangle_{t}}}=\frac{2 D}{1+D^{2}}
$$

Finally, we introduce an exogenous noise in the dynamics of the intensities 


$$
\begin{aligned}
& \mathrm{d} P_{t}^{i, \delta}=\Delta P_{i} \sqrt{\delta}\left(\mathrm{d} N_{t / \delta}^{i,+}-\mathrm{d} N_{t / \delta}^{i,-}\right) \\
& \lambda_{t}^{i, \pm}=\mu+\int_{0}^{t} \phi(t-s) \mathrm{d} N_{s}^{i, \mp}+\int_{0}^{t} \psi(t-s) \mathrm{d} N_{s}^{j, \pm}+M_{t}^{ \pm}
\end{aligned}
$$

We assume that there exists two random processes $\left(M_{t}^{0, \pm}, t \geq 0\right)$ such that

$$
\lim _{\delta \rightarrow 0} \sqrt{\delta} \int_{0}^{t / \delta} M_{s}^{ \pm} \mathrm{d} s=M_{t}^{0, \pm}
$$

and $\left\langle M^{0, \pm}\right\rangle_{t}<\infty$. Then the diffusive limit reads

$$
\begin{aligned}
\mathrm{d} P_{t}^{i, 0}=\Delta P_{i} \cdot C\left(\sigma\left(\mathrm{d} B_{t}^{i}+D \cdot \mathrm{d} B_{t}^{j}\right)+E \cdot \mathrm{d} M_{t}^{0}\right) \\
\sigma:=\sqrt{\frac{2 \mu}{1-(\|\phi\|+\|\psi\|)}} \\
C:=\frac{1+\|\phi\|}{(1+\|\phi\|)^{2}-\|\psi\|^{2}} \\
D:=\frac{\|\psi\|}{1+\|\phi\|} \\
E:=\frac{1+\|\phi\|+\|\psi\|}{1+\|\phi\|} \\
M_{t}^{0}:=M_{t}^{0,+}-M_{t}^{0,-}
\end{aligned}
$$

